\title{
Stochastic parallel transport on the d-dimensional Torus
}

\author{
Ana Bela Cruzeiro \\ Departamento de Matemática, Instituto Superior Técnico, \\ Universidade Técnica de Lisboa, \\ Av. Rovisco Pais, 1049-001 Lisboa, Portugal, \\ and \\ Grupo de Física-Matemática, Universidade de Lisboa \\ Av. Prof. Gama Pinto, 2, 1649-003 Lisboa, Portugal \\ E-mail: abcruz@math.ist.utl.pt \\ Paul Malliavin \\ 10 Rue S. Louis en-l'Ile, 75004 Paris, France
}

In Ref. $2 \mathrm{~V}$. Arnold has shown that the Euler flow can be identified with a geodesic on the group $G$ of volume preserving diffeomorphisms with respect to the $L^{2}$ metric. Following this approach, the geometry of $G$ plays a fundamental role in hydrodynamics and is important for instance in the study of the stability of the fluids motion. It has been developed by many authors, one of the first being Ref. 5, see also Ref. 3 and references therein.

In this paper we consider the $d$-dimensional torus as the underlying manifold, we compute the constants of structure and the Christoffel symbols of the corresponding group of volume preserving $L^{2}$ diffeomorphisms.

Using infinite dimensional stochastic analysis we construct the stochastic parallel transport on $G$ along Brownian paths where some weights on the Fourier modes are considered. Then there is a matrix which describes the energy transfer between modes: its exact computation in the two dimensional case has been done in Ref. 4, where a machinery already developed in Ref. 1 in the context of the Virasoro algebra was used. Here, for the $d$-dimensional case, we prove the existence of such matrix and establish some qualitative estimates.

Keywords: Stochastic parallel transport; geometry of diffeomorphisms group

\section{Basis of Lie algebra of vector fields with vanishing divergence}

We shall denote by $G$ the group of measure preserving diffeomeophisms of the torus $T^{d}$ and by $\mathcal{G}$ its Lie algebra, consisting of real vector fields on $T^{d}$ with vanishing divergence. 
We look for an $L^{2}$-orthonormal basis of complex vector fields with vanishing divergence. We use the key fact of the invariance of this space by the translation operator:

$$
\left[\left(\tau_{\varphi}\right)_{*} Y\right](\theta):=Y(\theta-\varphi) .
$$

Define

$$
Y^{n}:=\frac{1}{(2 \pi)^{d}} \int_{T^{d}}\left[\left(\tau_{\varphi}\right)_{*} Y\right] \quad \exp (i n . \varphi) d \varphi, \quad n=\left(n_{1}, \ldots, n_{d}\right) \in Z^{d} .
$$

Then $Y^{n}$ has a vanishing divergence; furthermore

$$
\left(\left(\tau_{\varphi_{0}}\right)_{*} Y^{n}\right)(\theta)=\frac{1}{(2 \pi)^{d}} \int_{T^{d}} Y\left(\theta-\varphi-\varphi_{0}\right) \quad \exp (i n . \varphi) d \varphi .
$$

Make the change of variable $\varphi^{\prime}-\varphi_{0}=\varphi$; then

$$
\left.\left(\left(\tau_{\varphi_{0}}\right)_{*} Y^{n}\right)(\theta)=\exp \left(-i n . \varphi_{0}\right) Y^{n}\right)(\theta) .
$$

Considering this identity at $\theta=0$ we find $Y^{n}\left(-\varphi_{0}\right)=\exp \left(-i n . \varphi_{0}\right) Y^{n}(0)$. Therefore we can look for a basis of vector fields with vanishing divergence of the form

$$
z_{n} \exp (i n . \theta), \quad z_{n} \text { is a fixed vector in } R^{d} .
$$

As we have $\operatorname{div}\left(z_{n} \exp (i n . \theta)\right)=i$ n. $z_{n} \exp (i n . \theta)$, we get

Proposition 1.1. The vector fields of the form

$$
z_{k} \exp (i k . \theta), \quad k . z_{k}=0
$$

generate the vector space of complex vector fields with zero divergence, and vector fields asssociated to two distinct values of $k$ are orthogonal.

To find an orthonormal basis we have to find for each $k$ an orthonormal basis of the space $V_{k}:=\left\{z \in C^{d}: k . z=0\right\}$; define

$$
\mathcal{E}_{k}:=\left\{x \in R^{d}: \quad k \cdot x=0\right\}, \text { then } \mathcal{E}_{-k}=\mathcal{E}_{k} ;
$$

and $V_{k}=\mathcal{E}_{k} \otimes C$. We obtain the wanted orthonormal basis by picking, for $\forall k \neq 0$, an orthonormal basis $\epsilon_{k}^{1}, \ldots, \epsilon_{k}^{d-1}$ of each $\mathcal{E}_{k}$; we make the convention to take

$$
\epsilon_{-k}^{\alpha}:=\epsilon_{k}^{\alpha} .
$$

Proposition 1.2. Denote $\tilde{Z}^{d}$ a subset of $Z^{d}$ such that each equivalence class of the equivalence relation on $Z^{d}$ defined by $k \simeq k^{\prime}$ if $k+k^{\prime}=0$ has a 
unique repesentative in $\tilde{Z}^{d}$; denote $\mathcal{H}_{r}$ the Hilbert space of square integrable real vector fields on $T^{d}$ with a vanishing divergence, then

$$
\left\{\epsilon_{k}^{\alpha} \cos k \cdot \theta, \quad \epsilon_{k}^{\alpha} \sin k \cdot \theta,\right\}_{k \in \tilde{Z}^{d}, \alpha \in[1, d-1]}
$$

constitute an orthonormal basis of $\mathcal{H}_{r}$.

Proof. We have

$$
Y=\sum_{k} z_{k} \times \exp (i k \cdot \theta), \quad z_{k} \in \mathcal{E}_{k}, \text { with the reality condition } z_{-k}=\bar{z}_{k}
$$

and $\|Y\|_{L^{2}}^{2}=\sum_{k}\left\|z_{k}\right\|_{R^{d}}^{2}$.

We can write the complex series $\left(1.3_{a}\right)$ as a real series by grouping terms:

$$
Y=\sum_{k \in \tilde{Z}^{d}}\left(z_{k} \times \exp (i k \cdot \theta)+\bar{z}_{k} \times \exp (-i k \cdot \theta)\right), \quad z_{0} \in R^{d}, \quad z_{k} \in \mathcal{E}_{k} \otimes C .
$$

For $k \in \tilde{Z}^{d}$, writing $z_{k}=\alpha_{k}-i \beta_{k}, \quad \alpha_{k}, \beta_{k} \in \mathcal{E}_{k}$, we get

$$
Y=\sum_{k \in \tilde{Z}^{d}}\left(\alpha_{k} \times \cos (i k . \theta)+\beta_{k} \times \sin (i k . \theta)\right), \quad \alpha_{0} \in R^{d}, \quad \alpha_{k}, \beta_{k} \in \mathcal{E}_{k} .
$$

We want to write automatically the passage from the representation $\left(1.3_{a}\right)$ to the representation $\left(1.3_{c}\right)$. We shall formalize the following elementary Euler identities

$\cos k \cdot \theta=\frac{1}{2}(\exp (i k \cdot \theta)+\exp (-i k \cdot \theta)), \sin k \cdot \theta=\frac{1}{2 i}(\exp (i k \cdot \theta)-\exp (-i k \cdot \theta))$.

Consider the group $\varsigma$ generated by the symmetry $\tau: k \mapsto-k$ on $Z^{d}$; then $\varsigma$ is a group of order 2. Denote $\chi$ the character on $\varsigma$ which takes the value -1 on $\tau$ and the value 1 on the identity. The powers of $\chi$ generate a group of order two; then there exists a coupling of duality $\varsigma \times \hat{\varsigma} \mapsto\{1,-1\}$, coupling denoted $\langle *, *\rangle$, where $\hat{\varsigma}$ is the dual group of $\varsigma$. Given a function $a$ defined on $Z^{d}$, its $\varsigma$-Fourier transform is defined as

$$
\hat{\sigma}^{\hat{\sigma}} a_{k}=\frac{1}{2} \sum_{\sigma \in \varsigma} a_{\sigma(k)} \times<\sigma, \hat{\sigma}>, \quad k \in \tilde{Z}^{d} .
$$

Starting from $\left(1.3_{c}\right)$ we define a function $\psi$ on $\hat{\varsigma} \times \tilde{Z}^{d}$ by

$$
\alpha_{k}=: \psi_{k}(e), \quad \beta_{k}=: \psi_{k}(\chi), \quad k \in \tilde{Z}^{d},
$$


then we get the universal formula describing the passage from $\left(1.3_{a}\right)$ to $\left(1.3_{c}\right)$

$$
\psi_{k}(\hat{\sigma})=\frac{1}{\sqrt{<\tau, \gamma>}} \times{ }^{\hat{\sigma}} z_{k}, \quad k \in \tilde{Z}^{d} .
$$

If we apply $\left(1.3_{e}\right)$ to the function $\exp (i k \cdot \theta)$ we find back the Euler identities $\left(1.3_{d}\right)$.

\section{Constants of structure of the Lie algebra and Christofell symbols}

The bracket of complex vector fields has the following expression:

$\left[z_{k} \times \exp (i k . \theta), z_{s} \times \exp (i s . \theta)\right]=\left(\left(z_{k} . s\right) z_{s}-\left(z_{s} . k\right) z_{k}\right) \times \exp (i(k+s) \cdot \theta)$;

as $\left(z_{k} . s\right)(k+s) . z_{s}-\left(z_{s} . k\right)(k+s) . z_{k}=0,\left(\left(z_{k} . s\right) z_{s}-\left(z_{s} . k\right) z_{k}\right) \in \mathcal{E}_{k+s}$.

Let

$$
\begin{gathered}
b_{k, s}:\left(\mathcal{E}_{k} \otimes C\right) \times\left(\mathcal{E}_{s} \otimes C\right) \mapsto \mathcal{E}_{k+s} \otimes C \text { be defined by } \\
b_{k, s}\left(z_{k}, z_{s}\right)=\left(\left(z_{k} . s\right) z_{s}-\left(z_{s} \cdot k\right) z_{k}\right) ; \text { hence } \\
{\left[z_{k} \times \exp (i k . \theta), z_{s} \times \exp (i s . \theta)\right]=b_{k, s}\left(z_{k}, z_{s}\right) \times \exp (i(k+s) \cdot \theta) .\left(2.1_{a}\right)}
\end{gathered}
$$

Remark that, granted the identification $\left(1.1_{b}\right)$, we have

$$
b_{-k,-l}=-b_{k, l} .
$$

The function $b_{k, l}$ is defined on $\mathcal{E}_{k} \times \mathcal{E}_{l}$ and takes its values in $\mathcal{E}_{k+l}$. In the orthonormal basis $\epsilon_{k}^{\alpha}, \epsilon_{l}^{\beta}, \epsilon_{k+s}^{\gamma}$ it is expressed as

$$
\left[b_{k, l}\right]_{\alpha, \beta}^{\gamma} \text { or more intrincally } b_{k, l} \in\left(\mathcal{E}_{k} \otimes \mathcal{E}_{l} \otimes \mathcal{E}_{k+l}\right) \otimes C,
$$

this last indentification being possible granted the euclidean structure of the $\mathcal{E}_{*}$.

Let $Y, Y^{\prime}$ be two real vector fields

$$
\begin{aligned}
& Y=\sum_{k \in \tilde{Z}^{d}}\left(z_{k} \times \exp (i k . \theta)+\bar{z}_{k} \times \exp (-i k . \theta)\right), \\
& Y^{\prime}=\sum_{s \in \tilde{Z}^{d}}\left(z_{s}^{\prime} \times \exp (i s . \theta)+\bar{z}_{s}^{\prime} \times \exp (-i s . \theta)\right),
\end{aligned}
$$




$$
\begin{gathered}
{\left[Y, Y^{\prime}\right]=\sum_{k, s \in \tilde{Z}^{d}} A_{k, s} \text { where }} \\
A_{k, s}:=\left[z_{k} \times \exp (i k . \theta)+\bar{z}_{k} \times \exp (-i k \cdot \theta), z_{s}^{\prime} \times \exp (i s . \theta)+\bar{z}_{s}^{\prime} \times \exp (-i s \cdot \theta)\right] \\
=b_{k, s}\left(z_{k}, z_{s}^{\prime}\right) \times i \exp (i(k+s) \cdot \theta)+b_{-k, s}\left(\bar{z}_{k}, z_{s}^{\prime}\right) \times i \exp (i(-k+s) \cdot \theta) \\
+b_{k,-s}\left(z_{k}, \bar{z}_{s}^{\prime}\right) \times i \exp (i(k-s) \cdot \theta)+b_{-k,-s}\left(\bar{z}_{k}, \bar{z}_{s}^{\prime}\right) \times i \exp (-i(k+s) \cdot \theta)
\end{gathered}
$$

Denote by $\delta$ the Kronecker symbol and define the constants of structure of the complex Lie algebra $\mathcal{H}$ by

$$
c_{k, s}^{r}=b_{k, s} \delta_{k+s}^{r}
$$

Previously the component of a vector was depending only upon indices $k \in Z^{d}$. Now the constants of structure depend upon three indices; the natural group of symmetry is $\varsigma^{3}$, group which has for dual group $\hat{\varsigma}^{3}$, the coupling being given by

$$
<\sigma, \hat{\sigma}>=\prod_{i=1}^{3}<\sigma_{i}, \hat{\sigma}_{i}>
$$

Define

$$
\begin{gathered}
{ }^{\hat{\sigma}} c_{k, s}^{r}=\frac{1}{8 \sqrt{<\tau, \gamma>}} \sum_{\sigma \in \varsigma^{3}} c_{\sigma_{1}(k), \sigma_{2}(s)}^{\sigma_{3}(r)}<\sigma, \hat{\sigma}>, \\
\tau=\left(\tau_{1}, \tau_{2}, \tau_{3}\right), \quad k, s, r \in \tilde{Z}^{d}, \quad \hat{\sigma} \in \hat{\varsigma}^{3} .
\end{gathered}
$$

Remark 2.1. We have $c_{\sigma_{1}(k), \sigma_{2}(s)}^{\sigma_{3}(r)} \in \mathcal{E}_{\sigma_{1}(k)} \otimes \mathcal{E}_{\sigma_{2}(s)} \otimes \mathcal{E}_{\sigma_{3}(r)} \otimes C$ which by $\left(1.1_{b}\right)$ is equal to $\mathcal{E}_{k} \otimes \mathcal{E}_{s} \otimes \mathcal{E}_{r} \otimes C$; therefore all the elements of the sum (2.2) belong to the same vector space.

Theorem 2.1. Define a function $\psi$ giving the expression of the bracket in the basis

$x_{k} \cos k \cdot \theta, y_{k} \sin k . \theta, \quad k \in \tilde{Z}^{d}, \quad x_{k}, y_{k} \in \mathcal{E}_{k}$ as

$\psi_{k, s}^{r}(1,1,1)$ is the term which gives the contribution of the terms in $\cos k \cdot \theta,, \cos s . \theta$ on the component on $\cos r \theta ; \psi_{k, s}(1,1, \chi)$ the component of the same term on $\sin r \theta$ and so on. Then we have the universal formula

$$
\psi_{k, s}^{r}(\hat{\sigma})={ }^{\hat{\sigma}} c_{k, s}^{r} .
$$


Proof. We define an induction on the number of components. We have proved the universal formula when we have a single component. Assume that we have proved it for tensors with a number of indices $<p$. Consider a tensor with p indices. Fixing the last index we obtain a tensor with $(p-1)$ indices for which the universal formula holds true; fixing the first $(p-1)$ components we obtain a vector for which the universal formula holds true; finally the universal formula stays stable by cartesian product.

\section{Christofell symbols}

The Christofell symbol in the complex basis are defined in tems of the structural constant

$$
2 \Gamma_{k, s}^{l}:=c_{k, s}^{l}-c_{s, l}^{k}+c_{l, k}^{s}=b_{k, s} \delta_{k+s}^{l}-b_{s, l} \delta_{s+l}^{k}+b_{l, k} \delta_{k+l}^{s}
$$

Remark 2.2. If $k l s \neq 0$ at most a single Kronecker symbol does not vanish and in the sum $\left(2.4_{a}\right)$ at most one term is different from zero; for this reason we have not to worry about adding objects which belong to different vector spaces. To avoid to check constantly facts of this nature we shall use the canonical injections $j_{k}: \mathcal{E}_{k} \mapsto R^{d}$, which induce a canonical injection $j_{k} \otimes j_{s} \otimes j_{l}: \mathcal{E}_{k} \otimes \mathcal{E}_{s} \otimes \mathcal{E}_{l} \mapsto R^{d} \otimes R^{d} \otimes R^{d}$; all the considered objects will belong to the fixed vector space $R^{d} \otimes R^{d} \otimes R^{d}$.

We obtain the Christofell symbols in the real basis by applying the universal formula $(2.2)$ :

$$
\begin{gathered}
{\left[{ }^{\hat{\sigma}} \Gamma\right]_{k, s}^{l}:=\frac{1}{8 \sqrt{<\tau, \gamma>}} \sum_{\sigma \in G^{3}} \Gamma_{\sigma_{1}(k), \sigma_{2}(s)}^{\sigma_{3}(l)} \times<\sigma, \hat{\sigma}>} \\
<\sigma, \hat{\sigma}>:=\prod_{i=1}^{3}<\sigma_{i}, \quad \hat{\sigma}_{i}>
\end{gathered}
$$

where $k, s, l \in \tilde{Z}^{d}$.

Proposition 2.1. For every $\gamma \in \hat{\sigma}^{3}$ we have $\left[{ }^{\hat{\sigma}} \Gamma\right]_{k, s}^{l} \in \mathcal{E}_{k} \otimes \mathcal{E}_{s} \otimes \mathcal{E}_{l}$.

In the usual theory Christofell coefficients are scalar; to realize this situation we have to pick a basis $\epsilon_{k}^{\alpha}, \alpha \in[1, d-1]$ of each $\mathcal{E}_{k}$; then the coefficient $\left[{ }^{\gamma} \Gamma\right]_{k, s}^{l}$ will give rise to $(d-1)^{2}$ scalar coefficients. We prefer to avoid this explicit computation by introducing the following vector valued tensor calculus. We consider the vector bundle $\mathcal{F}$ of basis $\tilde{Z}^{d}$ defined as

$$
\mathcal{F}:=\bigcup_{k \in \tilde{Z}^{d}} \mathcal{E}_{k} \text { and its dual bundle defined as } \mathcal{F}:=\bigcup_{k \in \tilde{Z}^{d}} \mathcal{E}_{k}^{*} ;
$$

of course the euclidean structure of $\mathcal{E}_{k}$ defines an identification of $\left(\mathcal{E}_{k}^{*}\right)^{*}$ with $\mathcal{E}_{k}$. In tensor calculus we shall forget this identification. A tensor $q$-times 
covariant and $p$-times contravariant is by definition a section of the vector bundle $\left[\mathcal{F}^{*}\right]^{\otimes q} \otimes[\mathcal{F}]^{\otimes p}$

Proposition 2.2. Fix $\hat{\sigma} \in \hat{\varsigma}^{3}$, then we can consider $[\hat{\sigma} \Gamma]_{*, *}^{*}$ as a tensor 2-times covariant and 1-times contravariant.

Proof. Use Prop. 2.1 and the identification between $\left[\mathcal{E}_{k}\right]^{*}$ and $\mathcal{E}_{k}$.

\section{Tensorial contraction}

Given $\xi^{*}$ a contravariant tensor an $\eta_{*}$ a covariant tensor their contraction is

$$
\sum_{k \in \tilde{Z}^{d}} \operatorname{trace} \xi^{k} \otimes \eta_{k}:=\sum_{k \in \tilde{Z}^{d}}<\xi^{k}, \eta_{k}>
$$

We can contract two contravariant (resp. covariant) indices using the identification of $\mathcal{E}_{k}$ with $\mathcal{E}_{k}$ granted the underlying euclidean metric. We shall emphasize the use of this euclidean structure by the notation

$$
\sum_{k \in \tilde{Z}^{d}} \operatorname{trace}_{k, k} \xi^{k} \otimes \eta^{k}=\sum_{k \in \tilde{Z}^{d}}\left(\xi^{k} \mid \eta^{k}\right)_{\mathcal{E}_{k}}
$$

Proposition 2.3. Fix $k \in Z^{d}$ and fix $z \in \mathcal{E}_{k}$; denote $\boldsymbol{\Gamma}(z)$ the corresponding operator on $\mathcal{F}$. Then $\boldsymbol{\Gamma}(z)$ is antisymmetric operator.

Proof. Choose $z_{*}, z_{*}^{\prime}, z_{*}^{\prime \prime}$ such that $z_{-q}=\bar{z}_{q}, z_{-s}^{\prime}=\bar{z}_{q}^{\prime}, \quad z_{-q}^{\prime \prime}=\bar{z}_{q}$. Define the euclidean scalar product on $\mathcal{E}_{*} \otimes C$ by the algebraic prolongation of the euclidean scalar product on $\mathcal{E}_{*}$ : we obtain a scalar product which is $C$-linear relatively to the second factor. Using formula $\left(2.4_{a}\right)$, introduce the expression

$$
\sum_{k, s}\left(b_{k, s}\left(z_{k}, z_{s}^{\prime}\right) \mid z_{k+s}^{\prime \prime}\right)-\left(b_{s, k-s}\left(z_{s}^{\prime}, z_{k-s}^{\prime \prime}\right) \mid z_{k}\right)+\left(b_{s-k, k}\left(z_{s-k}^{\prime \prime}, z_{k}\right) \mid z_{s}^{\prime}\right) .
$$

Fix $z$ and show the antisymmetry in $z^{\prime}, z^{\prime \prime}$; firstly

$$
b_{s, k-s}\left(z_{s}^{\prime}, z_{k-s}^{\prime \prime}\right)=-b_{k-s, s}\left(z_{k-s}^{\prime \prime}, z_{s}^{\prime}\right)
$$

according to the expression

$$
b_{k, l}\left(z_{k}, z_{s}\right)=\left(z_{k} . s\right) z_{s}-\left(z_{s} . k\right) z_{k}
$$

therefore the antisymmetry of the middle term of $\left(2.5_{a}\right)$ is obtained. 
In the third term of $\left(2.5_{a}\right)$ making the change of index of summation $(s-k) \mapsto q \simeq s$, we obtain that the contribution of the first and third term of $\left(2.5_{a}\right)$ can be written as

$$
\sum_{k, s}\left(b_{k, s}\left(z_{k}, z_{s}^{\prime}\right) \mid z_{k+s}^{\prime \prime}\right)+\left(b_{s, k}\left(z_{s}^{\prime \prime}, z_{k}\right) \mid z_{k+s}^{\prime}\right)
$$

On the other hand,

$$
\begin{aligned}
& \left(b_{k, s}\left(z_{k}, z_{s}^{\prime}\right) \mid z_{k+s}^{\prime \prime}\right)+\left(b_{s, k}\left(z_{s}^{\prime \prime}, z_{k}\right) \mid z_{k+s}^{\prime}\right) \\
= & \left(z_{k} . s\right)\left(z_{s}^{\prime} \mid z_{k+s}^{\prime \prime}\right)-\left(z_{s}^{\prime} \cdot k\right)\left(z_{k} \mid z_{k+s}^{\prime \prime}\right)- \\
& \left(z_{k} . s\right)\left(z_{s}^{\prime \prime} \mid z_{k+s}^{\prime}\right)+\left(z_{s}^{\prime \prime} . k\right)\left(z_{k} \mid z_{k+s}^{\prime}\right) \\
= & -\left(z_{s}^{\prime} . k\right)\left(z_{k} \mid z_{k+s}^{\prime \prime}\right)+\left(z_{s}^{\prime \prime} \cdot k\right)\left(z_{k} \mid z_{k+s}^{\prime}\right),
\end{aligned}
$$

expression which is obviously antsymmetric in $z^{\prime}, z^{\prime \prime}$.

Corollary 2.1. For fixed $k$ the matrices

$$
{ }^{\hat{\sigma}} \Gamma_{k, *}^{*} \text { are antisymmetric } \forall \hat{\sigma} \in \hat{\varsigma}^{3} \text {. }
$$

Proof. As ${ }^{\gamma} \Gamma_{k, *}^{*}$ is obtained by averaging an antisymmetric operator, it is antisymmetric.

Notation 2.1. Given $\xi^{k}$ a contravariant tensor, we denote the following antisymmetric operator

$$
\sum_{k}^{\hat{\sigma}} \Gamma_{k, *}^{*} \xi^{k}=:{ }^{\hat{\sigma}} \boldsymbol{\Gamma}(\xi)_{*}^{*} .
$$

Proposition 2.4. The component of the Christofell symbol on the three cosines type vanishes.

Proof. This component is equal to ${ }^{\hat{\sigma}_{0}} \Gamma$, where $\hat{\sigma}_{0}=(1,1,1)$; remark that $<\tau \sigma, \hat{\sigma}_{0}>=<\sigma, \hat{\sigma}_{0}>$ and that $\delta_{\tau(k), \tau(s)}^{\tau(l)}=\delta_{k, s}^{l}$. Therefore the sum of twenty four terms defining ${ }^{\sigma_{0}} \Gamma$ can be split in the sum of twelve terms each having in factor the following expression $\delta_{k^{\prime}, s^{\prime}}^{l^{\prime}} \times\left(b_{k^{\prime}, s^{\prime}}+b_{\tau\left(k^{\prime}\right), \tau\left(s^{\prime}\right)}\right)$ and $\left(2.1_{b}\right)$ finishes the proof.

\section{Stochastic parallel transport, symmetries of the noise}

Consider for each $k \in \tilde{Z}^{d}$ the complex brownian motion $\zeta_{k}(t)$ associated to the natural hermitian metric on $\mathcal{E}_{k} \otimes C$; all these brownian motion are taken to be independent on the system of relations

$$
\zeta_{-k}(t)=\bar{\zeta}_{k}(t) \text {, or in real terms } \zeta_{k}=x_{k}+i y_{k}(t), \quad k \in \tilde{Z}^{d},
$$


the $x_{k}, y_{k}$ being independent brownian motion on $\mathcal{E}_{k}$. Define

$$
\boldsymbol{\Gamma}\left(d x_{k}(t)\right)=\sum_{\gamma_{1}=1}^{\gamma} \boldsymbol{\Gamma}\left(d x_{k}(t)\right), \quad \boldsymbol{\Gamma}\left(d y_{k}(t)\right)=\sum_{\gamma_{1}=-1}^{\gamma} \boldsymbol{\Gamma}\left(d y_{k}(t)\right),
$$

where the sumation is taken on $\gamma=\left(\gamma_{1}, \gamma_{2}, \gamma_{3}\right) \in \hat{\varsigma}^{3}$, the first sum been restricted to those $\gamma$ for which $\gamma_{1}=1$ the second to those $\gamma$ for which $\gamma_{1}=-1$.

Choose a weight $\rho(k) \geq 0, \quad k \in \tilde{Z}^{d}$ and consider the $\mathcal{G}$ valued process

$$
p_{t}=\sum_{k \in \tilde{Z}^{d}} \rho(k)\left(x_{k}(t) \times \cos k . \theta+y_{k}(t) \times \sin k . \theta\right)
$$

Consider the Stratonovitch SDE,

$$
\begin{gathered}
d \psi_{t}=\left(\sum_{k \in \tilde{Z}^{d}} \rho(k)\left(\boldsymbol{\Gamma}\left(d x_{k}(t)\right)+\boldsymbol{\Gamma}\left(d y_{k}(t)\right)\right)\right) \circ \psi_{t}=\boldsymbol{\Gamma}\left(d p_{t}\right) \circ \psi_{t}, \\
\psi_{0}=\text { Identity. }
\end{gathered}
$$

As the $\boldsymbol{\Gamma}$ are antisymmetric operators this equation takes formally its values in the unitary group of $\mathcal{G}$; establishing this fact under mild assumptions on the weight $\rho$, will be the purpose of next four paragraphs.

The geometric meaning of (3.3) is to describe in terms of the algebraic parallelism inherited from the group structure of $G$ the Levi-Civita parallelism inherited from the Riemannian structure of $G$; for this reason we call (3.3) the equation of the stochastic parallel transport.

\section{Symmetries of the noise}

Denote as before by $G$ the group of measure preserving diffeomeophisms of the torus $T^{d}$ and by $\mathcal{G}$ its Lie algebra.

Proposition 3.1. Given $g \in G, z \in \mathcal{G}$, the adjoint action

$$
\left.z \mapsto \frac{d}{d \epsilon}\right|_{\epsilon=0} g \exp (\epsilon z) g^{-1},
$$

is the direct image $g_{*}(z)$ of the vector field $z$ by the diffeomorphism $g$.

Proof. Reference [6], page 210.

In particular the translation $\tau_{\varphi}: \theta \mapsto \theta+\varphi$ is a diffeomorphism whose Jacobian matrix is the Identity; therefore

$$
\left[\left(\tau_{\varphi}\right)_{*}(z)\right](\theta)=z(\theta-\varphi)
$$


The collection $\left(\tau_{\varphi}\right)_{*}, \quad \varphi \in T^{d}$, constitutes a unitary representation of $T^{d}$ on $\mathcal{G}$ which decomposes into irreducible components along the basis

$$
\cos k . \theta \otimes \mathcal{E}_{k}, \quad \sin k . \theta \otimes \mathcal{E}_{k},
$$

the action of $\left(\tau_{\varphi}\right)_{*}$ on $\mathcal{E}_{k} \oplus \mathcal{E}_{k}$ being the rotation

$$
\mathcal{D}_{k}(\varphi):=\left(\begin{array}{rr}
\cos k \varphi & -\sin k \varphi \\
\sin k \varphi & \cos k \varphi
\end{array}\right) .
$$

Furthermore $\tau_{\varphi}$ preserves the Lie algebra structure. The Christofell symbols are derived from the Hilbertian structure and from the bracket structure of $\mathcal{G}$. Therefore they commute with $\tau_{\varphi}$ in the sense that

$$
\left.\left(\tau_{\varphi}\right)_{*}[\Gamma(\xi)(\eta)]=\Gamma\left(\left(\tau_{\varphi}\right)_{*} \xi\right)\left[\left(\tau_{\varphi}\right)_{*} \eta\right)\right], \quad \xi, \eta \in \mathcal{G}
$$

or denoting by $\boldsymbol{\Gamma}(z)$ the antihermitian endomorphism of $\mathcal{G}$ defined by the Christofell symbols, we have

$$
\boldsymbol{\Gamma}\left(\left(\tau_{\varphi}\right)_{*}(z)\right)=\left(\tau_{\varphi}\right)_{*} \circ \boldsymbol{\Gamma}(z) \circ\left(\tau_{-\varphi}\right)_{*}
$$

Denote by $\operatorname{su}(\mathcal{G})$ the vector space of antisymmetric operators on the Hilbert space $\mathcal{G}$.

Proposition 3.2. Let $p_{t}$ the $\mathcal{G}$-valued process defined in $\left(3.2_{b}\right)$ denote $p^{\varphi}=:\left(\tau_{\varphi}\right)_{*} p$; then $p^{\varphi}$ and $p$ have the same law.

Proof. The rotation $\mathcal{D}_{k}(\phi)$ preserves in law the Brownian motion on $\mathcal{E}_{k} \otimes$ $\mathcal{E}_{k}$.

Corollary 3.1. The processes $\left(\tau_{\varphi}\right) \circ \psi_{t} \circ\left(\tau_{-\varphi}\right)$ and $\psi_{t}$ have the same law.

Proof. Denoting by $\psi_{t}^{p}$ the solution of (3.3) associated the noise $p_{t}$, we have

$$
\left(\tau_{\varphi}\right) \circ \psi_{t} \circ\left(\tau_{-\varphi}\right)=\psi_{t}^{p^{\varphi}}
$$

Definition 3.1. We say that an endomorphism $u$ of $\mathcal{G}$ is pseudo diagonal in the direct sum decomposition $\mathcal{G}=\bigoplus \mathcal{V}_{k}$ if the restriction of $u$ to $\mathcal{V}_{k}$ takes its values in $\mathcal{V}_{k}$; then $u$ is determined by a sequence $u_{k} \in \operatorname{End}\left(\mathcal{V}_{k}\right)$; we denote $u=\left[u_{k}\right], k \in \tilde{Z}^{d}$. 
Theorem 3.1. The Itô contraction describing the passage from the Stratonovitch equation (3.3) to its associated Itô form is an operator $\mathcal{B}$ which is pseudo diagonal in the direct sum decomposition

$$
\mathcal{G}=\bigoplus_{k \in \tilde{Z}^{d}}\left[\mathcal{E}_{k} \oplus \mathcal{E}_{k}\right]
$$

Furthermore the diagonal term on the component $\mathcal{E}_{k} \oplus \mathcal{E}_{k}$ is of the form $-\left[\lambda_{k}, \lambda_{k}\right]$ where $\lambda_{k}$ is a positive symmetric operator on $\mathcal{E}_{k}$.

Proof. Denote $\mathcal{E}_{k}^{1}$ (resp. $\mathcal{E}_{k}^{2}$ ) the first (resp. the second) component of the direct sum $\mathcal{E}_{k} \oplus \mathcal{E}_{k}$ and pick an orthonormal basis $\epsilon_{k}^{\alpha}$ of each $\mathcal{E}_{k}$. As we have two injections of $\mathcal{E}_{k} \mapsto \mathcal{E}_{k} \oplus \mathcal{E}_{k}$, the first having its image in the first factor of the direct sum, the second in the second factor; we denote again $\epsilon_{k}^{\alpha}$ the image by the first injection and $\eta_{k}^{\alpha}$ the image by the second injection; we get in this way an orthonormal basis of $\mathcal{E}_{k}^{i}, i=1,2$.

The Itô SDE has the shape

$$
\begin{aligned}
& d \psi_{t}^{p}=\left(\sum _ { k \in \tilde { Z } ^ { d } } \rho \left(\boldsymbol{\Gamma}\left(d x_{k}(t)\right)\right.\right.\left.+\boldsymbol{\Gamma}\left(d y_{k}(t)\right)\right)+ \\
&\left.\frac{\rho}{2} \boldsymbol{\Gamma}\left(d x_{k}\right) * \boldsymbol{\Gamma}\left(d x_{k}\right)+\frac{\rho}{2} \boldsymbol{\Gamma}\left(d y_{k}\right) * \boldsymbol{\Gamma}\left(d y_{k}\right)\right) \psi_{t} .
\end{aligned}
$$

Denote by $x_{k}^{\varphi}(t)=\left(\tau_{\varphi}\right)_{*} x_{k}(t), y_{k}^{\varphi}(t)=\left(\tau_{\varphi}\right)_{*} y_{k}(t)$; then $x_{k}^{\varphi}(t)$ has the same law as $x_{k}(t)$. Therefore

$$
\left(d x_{t}^{\varphi}\right) *\left(d x_{t}^{\varphi}\right)=\left(d x_{t}\right) *\left(d x_{t}\right)
$$

Using formula $\left(3.5_{b}\right)$,

$$
\boldsymbol{\Gamma}\left(d x_{t}^{\varphi}\right) * \boldsymbol{\Gamma}\left(d x_{t}^{\varphi}\right)=\left(\tau_{\varphi}\right)_{*} \circ \boldsymbol{\Gamma}(d x) * \boldsymbol{\Gamma}(d x) \circ\left(\tau_{-\varphi}\right)_{*}
$$

Denoting

$$
\mathcal{B} d t=\frac{1}{2} \sum_{k \in \tilde{Z}^{d}}[\rho(k)]^{2}\left(\boldsymbol{\Gamma}\left(d x_{k}\right) * \boldsymbol{\Gamma}\left(d x_{k}\right)+\boldsymbol{\Gamma}\left(d y_{k}\right) * \boldsymbol{\Gamma}\left(d y_{k}\right)\right)
$$

we have

$$
\mathcal{B}=\left(\tau_{\varphi}\right)_{*} \circ \mathcal{B} \circ\left(\tau_{-\varphi}\right)_{*}
$$

Lemma 3.1. An endomorphism of $\mathcal{G}$ satisfying $\left(3.6_{b}\right)$ is diagonal in the basis $\left(3.6_{a}\right)$. 
Proof. Pick $A_{s} \in \mathcal{E}_{s}^{1}, \quad A_{k} \in \mathcal{E}_{k}^{1}, \quad B_{s} \in \mathcal{E}_{s}^{2}, \quad B_{k} \in \mathcal{E}_{k}^{2}$ and consider the $2 \times 2$ matrix $M_{s, k}$

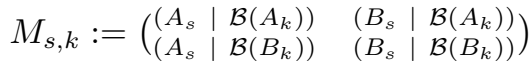

Then formula $\left(3.6_{c}\right)$ implies that

$$
M_{s, k}=\mathcal{D}_{s}(\varphi) \circ M_{s, k} \circ \mathcal{D}_{k}(-\varphi)
$$

Integrating this identity over $T^{d}$ we get

$$
(2 \pi)^{d} M_{s, k}=\int_{T^{d}} \mathcal{D}_{s}(\varphi) \circ M_{s, k} \circ \mathcal{D}_{k}(-\varphi) d \varphi_{1} \otimes d \varphi_{2}
$$

If we develop the product of matrices of the r.h.s. we find an expression of the form $(\cos s . \varphi)(\cos k . \varphi)$ which will have an integral equal to 0 if $s \neq k$.

As $\boldsymbol{\Gamma}(*)$ are antisymmetric we get that their square are negative symmetric operators.

As on $\mathcal{E}_{k} \oplus \mathcal{E}_{k}$, this symmetric operator commutes with the rotations, it decomposes into two identic copies on each component.

\section{Transfer energy matrix of the stochastic parallel transport}

A sequence of vectors with norm 1 of an Hilbert space can converge weakly to zero: this phenomena corresponds to a "dissipation of energy towards the higher modes". For the construction of the stochastic parallel transport it is essential to control this dissipation.

We fix $\xi_{0} \in \mathcal{G}$ and consider

$$
\xi_{t}^{p}:=\psi_{t}^{p}\left(\xi_{0}\right)
$$

Pick $A_{s} \in \mathcal{E}_{s}^{1}, \quad A_{k} \in \mathcal{E}_{k}^{1}, \quad B_{s} \in \mathcal{E}_{s}^{2}, \quad B_{k} \in \mathcal{E}_{k}^{2}$ and consider

$$
\begin{gathered}
\alpha_{s, k}^{1}\left(\xi_{0}\right)=E\left(\left(A_{s} \mid \xi_{t}^{p}\right) \times\left(A_{k} \mid \xi_{t}^{p}\right)\right), \quad \alpha_{s, k}^{2}\left(\xi_{0}\right)=E\left(\left(B_{s} \mid \xi_{t}^{p}\right) \times\left(B_{k} \mid \xi_{t}^{p}\right)\right), \\
\alpha_{s, k}^{3}\left(\xi_{0}\right)=E\left(\left(A_{s} \mid \xi_{t}^{p}\right) \times\left(B_{k} \mid \xi_{t}^{p}\right)\right)
\end{gathered}
$$

Theorem 4.1. Denote $\left(\tau_{\varphi}\right)_{*}\left(\xi_{0}\right):=\xi_{0}^{\varphi}$, then

$$
\int_{T^{d}} \alpha_{s, k}^{i}\left(\xi_{0}^{\varphi}\right) d \varphi=0, \quad s \neq k, i=1,2,3 .
$$


Proof. Using $\left(3.5_{b}\right)$ we get

$$
\boldsymbol{\Gamma}\left(p_{t}^{\varphi}\right)=\left(\tau_{\varphi}\right)_{*} \circ \boldsymbol{\Gamma}\left(p_{t}\right) \circ\left(\tau_{-\varphi}\right)_{*}
$$

or by exponentiating

$$
\begin{aligned}
& \psi_{t}^{p^{\varphi}}=\left(\tau_{\varphi}\right)_{*} \circ \psi_{t}^{p} \circ\left(\tau_{-\varphi}\right)_{*} \\
& \left(\tau_{-\varphi}\right)_{*} \circ \psi_{t}^{p^{\varphi}}=\psi_{t}^{p} \circ\left(\tau_{-\varphi}\right)_{*}
\end{aligned}
$$

Applying this identity to the vector $\xi_{0}$ and changing $\varphi$ in $-\varphi$ we get

$$
\left(\tau_{\varphi}\right)_{*} \circ \psi^{p_{t}^{-\varphi}}=\psi_{t}^{p}\left(\xi_{0}^{\varphi}\right)
$$

Therefore

$$
\alpha_{s, k}^{1}\left(\xi_{0}^{\varphi}\right)=E\left(\left(A_{s}^{-\varphi} \mid \xi_{t}^{p^{-\varphi}}\right) \times\left(A_{k}^{-\varphi} \mid \xi_{t}^{p^{-\varphi}}\right)\right)
$$

Using now the key fact that $p_{t}^{-\varphi}$ and $p_{t}$ have the same law, we obtain

$$
\alpha_{s, k}^{1}\left(\xi_{0}^{\varphi}\right)=E\left(\left(A_{s}^{-\varphi} \mid \xi_{t}^{p}\right) \times\left(A_{k}^{-\varphi} \mid \xi_{t}^{p}\right)\right)
$$

Using the identity $A_{k}^{-\varphi}=\cos (k . \varphi) \times A_{k}+\sin (k . \varphi) \times B_{k}$ and similar identities we get

$$
\begin{array}{r}
\alpha_{s, k}^{1}\left(\xi_{0}^{\varphi}\right)=\cos (k . \varphi) \cos (s . \varphi) \alpha_{s, k}^{1}\left(\xi^{0}\right)+\sin (k . \varphi) \sin (s . \varphi) \alpha_{s, k}^{2}\left(\xi^{0}\right) \\
+\cos (k . \varphi) \sin (s . \varphi) \alpha_{s, k}^{3}\left(\xi^{0}\right)+\sin (k . \varphi) \cos (s . \varphi) \alpha_{k, s}^{3}\left(\xi^{0}\right)
\end{array}
$$

Theorem 4.2. There exists a matrix $\mathcal{A}$ defined on $\tilde{Z}^{d} \times \tilde{Z}^{d}$ such that denoting

$$
u_{t}^{\xi_{0}}(k)=E\left(\sum_{\alpha=1}^{d-1}\left(\epsilon_{k}^{\alpha} \mid \xi_{t}^{p}\right)^{2}+\left(\eta_{k}^{\alpha} \mid \xi_{t}^{p}\right)^{2}\right)
$$

where the basis $\epsilon_{k}^{\alpha}, \eta_{k}^{\alpha}$, has been defined in the proof of Theorem 3.4, we have

$$
\frac{d u_{t}^{\xi_{0}}}{d t}=\mathcal{A} u_{t}^{\xi_{0}}
$$

Proof. We remark that $\left(4.2_{a}\right)$ implies that

$$
\begin{aligned}
& u_{t}^{\xi_{0}^{\varphi}}(k)=u_{t}^{\xi_{0}}(k) \quad \text { therefore } \\
& \frac{1}{(2 \pi)^{d}} \int_{T^{d}} u_{t}^{\xi_{0}^{\varphi}}(k) d \varphi=u_{t}^{\xi_{0}}(k)
\end{aligned}
$$


We can implement this fact by adding to the probability space generated by $p$ a random initial value $\xi_{0}^{\varphi}$ with a uniform repartition of the variable $\varphi$. By Itô calculus we can write

$$
\frac{d}{d t} \int_{T^{d}} u_{t}^{\xi_{0}^{\varphi}}(k)
$$

in terms of the

$$
\int_{T^{d}} \alpha_{s, k}^{i}\left(\xi_{0}^{\varphi}\right) d \varphi_{1} \otimes d \varphi_{2}
$$

expression which vanishes for $s \neq k$ by (4.1).

Corollary 4.1. We have

$$
u_{t}^{\xi_{0}}(k)=2 E\left(\sum_{\alpha=1}^{d-1}\left(\epsilon_{k}^{\alpha} \mid \xi_{t}^{p}\right)^{2}\right)
$$

Proof. Use the identity $\left(4.2_{c}\right)$ with $s=k$ and integrate in $\varphi$.

\section{Exact computation of the energy transfer matrix for $d=2$}

We shall present in section 6 indirect qualitative estimation of the energy transfer matrix valid in any dimension $d \geq 2$. In this section we recall the full computation from scratch of the transfer energy matrix in the case $d=2$ which was obtained in Ref. 4 . These computations give also another proof of the orthogonality results proved by symmetry arguments in section 4.

When $d=2$ the space $\mathcal{E}_{k}$ is of dimension 1 ; we take for orthonormal basis of $\mathcal{E}_{k}$ the vector $\left(\frac{k_{2}}{|k|},-\frac{k_{1}}{|k|}\right)$. We get for orthonormal basis (for the $L^{2}$ metric) of vector fields with vanishing divergence on the torus, firstly the two constant vector fields and

$$
\begin{aligned}
& \left.A_{k}=\frac{1}{|k|}\left[\left(k_{2} \cos k \cdot \theta\right) \partial_{1}-\left(k_{1} \cos k \cdot \theta\right) \partial_{2}\right)\right] \\
& \left.B_{k}=\frac{1}{|k|}\left[\left(k_{2} \sin k \cdot \theta\right) \partial_{1}-\left(k_{1} \sin k \cdot \theta\right) \partial_{2}\right)\right]
\end{aligned}
$$

where $k=\left(k_{1}, k_{2}\right) \in \tilde{Z}^{2}$, with $|k| \neq 0$, and where $k . \theta=k_{1} \theta_{1}+k_{2} \theta_{2}$. 
For $k, l \in \tilde{Z}^{2}, \quad k, l \neq(0,0)$, define on $\tilde{Z}^{2}$ the following functions

$$
\begin{gathered}
\left.\alpha_{k, l}:=\frac{1}{4|k||l||k+l|}\left(|l+k|^{2}-|k|^{2}+|l|^{2}\right)\right)=\frac{1}{2|k||l||k+l|}((l \mid l+k)) \\
\left.\beta_{k, l}:=\alpha_{-k, l}=\frac{1}{4|k||l||k-l|}\left(|l-k|^{2}-|k|^{2}+|l|^{2}\right)\right)=\frac{1}{2|k||l||k-l|}(l \mid(l-k)) \\
{[k, l]=k_{1} l_{2}-k_{2} l_{1} .}
\end{gathered}
$$

The brackets of the above mentionned vector fields are given in the following

Theorem 5.1 (Ref. 4). The following expressions hold

$$
\begin{gathered}
{\left[A_{k}, A_{l}\right]=\frac{[k, l]}{2|k||l|}\left(|k+l| B_{k+l}+|k-l| B_{k-l}\right)} \\
{\left[B_{k}, B_{l}\right]=-\frac{[k, l]}{2|k||l|}\left(|k+l| B_{k+l}-|k-l| B_{k-l}\right)} \\
{\left[A_{k}, B_{l}\right]=-\frac{[k, l]}{2|k||l|}\left(|k+l| A_{k+l}-|k-l| A_{k-l}\right)}
\end{gathered}
$$

Concerning the Christoffel symbols,

Theorem 5.2 (Ref. 4). The Cristoffel symbols are

$$
\begin{aligned}
\Gamma_{A_{k}, A_{l}} & =[k, l]\left(\alpha_{k, l} B_{k+l}+\beta_{k, l} B_{k-l}\right) \\
\Gamma_{B_{k}, B_{l}} & =[k, l]\left(-\alpha_{k, l} B_{k+l}+\beta_{k, l} B_{k-l}\right) \\
\Gamma_{A_{k}, B_{l}} & =[k, l]\left(-\alpha_{k, l} A_{k+l}+\beta_{k, l} A_{k-l}\right) \\
\Gamma_{B_{k}, A_{l}} & =[k, l]\left(-\alpha_{k, l} A_{k+l}-\beta_{k, l} A_{k-l}\right)
\end{aligned}
$$

In this two-dimensional case the equation for the stochastic parallel transport reads

$$
d \psi_{t}=-\left(\sum_{k \neq 0} \rho(k) \Gamma_{A_{k}, .} \text { od } x^{k}+\rho(k) \Gamma_{B_{k}, .} o d y^{k}\right) \psi_{t}
$$

with independent Brownian motions in all components, $\psi(0)=i d$.

We remark the difference with respect to Ref. 4 of the constants appearing in $\left(5.1_{a}\right)$ and $\left(5.1_{b}\right)$ : taking indices in $\tilde{Z}^{2}$, only half of the quantities computed in Ref. 4, Theorem 3.1, have to be considered. 
Theorem 5.3 (Ref. 4). The coefficient of the transfer energy matrix are given by

$$
\begin{aligned}
& \mathcal{A}_{l}^{l}=-2 \sum_{k}[\rho(k)]^{2}[l, k]^{2} \times\left(\beta_{k, k-l} \beta_{k, l}+\alpha_{k,-k-l} \alpha_{k, l}\right) \\
& \mathcal{A}_{s}^{l}=2 \sum_{k}[\rho(k)]^{2}[l, k]^{2} \times\left(\alpha_{k, l-k}^{2} \delta_{s}^{l-k}+\beta_{k, l+k}^{2} \delta_{s}^{l+k}\right)
\end{aligned}
$$

The matrix $\mathcal{A}$ is symmetric, the non diagonal terms are positive, the diagonal terms are negative, the sum of the coefficients on each column vanishes.

Remark 5.1. Notice that

$$
\begin{gathered}
\beta_{k, k-l} \beta_{k, l}=\alpha_{k, l-k}^{2}=\frac{(l \mid l-k)^{2}}{4|k|^{2}|l|^{2}|l-k|^{2}} \\
\alpha_{k,-k-l} \alpha_{k, l}=\beta_{k, l+k}^{2}=\frac{(l \mid l+k)^{2}}{4|k|^{2}|l|^{2}|l+k|^{2}}
\end{gathered}
$$

Corollary 5.1. Denote $\chi_{k, l}$ the angle between the vectors $k$ and $l$; then

$$
\begin{gathered}
\left|\mathcal{A}_{l}^{l}\right|=\frac{|l|^{2}}{8} \sum_{k \in \tilde{Z}^{2}}\left(1-\cos 4 \chi_{k, l}\right) \times[\rho(k)]^{2} \\
\sum_{k}[\rho(k)]^{2}[l, k]^{2} \times k\left(\alpha_{k, l-k}^{2}-\beta_{k, l+k}^{2}\right)=0
\end{gathered}
$$

If $\rho(k)$ depends only upon $|k|$

$$
\left|\mathcal{A}_{l}^{l}\right| \simeq c|l|^{2}, \quad 4 c:=\sum_{k \in \tilde{Z}^{2}}[\rho(k)]^{2}
$$

\section{Qualitative estimation of the energy transfert matrix for $\mathrm{d}>2$}

We propose ourselves to obtain in dimension greater than 2 the estimates (5.2).

We interpreted the ${ }^{\gamma} \Gamma_{*, s}^{l}$ as defining operators $\mathcal{E}_{1} \rightarrow \mathcal{E}_{l}$, the euclidean structure of the $\mathcal{E}_{j}$ makes possible to define their adjoint; then

$$
\operatorname{trace}\left({ }^{\gamma} \Gamma_{*, s}^{l}\right)\left({ }^{\gamma} \Gamma_{*, s}^{l}\right)^{*}
$$

is well defined and is equal to the HS norm (Hilbert-Schmidt norm). 
Theorem 6.1. The diagonal terms of the matrix $\mathcal{A}$ are expressed by the diagonal terms of the diagonal matrix $\mathcal{B}$ defined in $\left(3.6_{a}\right)$,

$$
\mathcal{A}_{l}^{l}=-2 \text { trace }\left(\lambda_{k}\right)
$$

and, as a consequence, are negative; the non diagonal terms are given by

$$
\mathcal{A}_{s}^{l}=\sum_{k \in \tilde{Z}^{d}}[\rho(k)]^{2}\left|\sum_{\gamma_{1}=1}^{\gamma} \Gamma_{k, s}^{l}\right|_{H S}^{2}+\sum_{k \in \tilde{Z}^{d}}[\rho(k)]^{2}\left|\sum_{\gamma_{1}=-1}{ }^{\gamma} \Gamma_{k, s}^{l}\right|_{H S}^{2}, \quad l \neq s ;
$$

The non diagonal terms are positive. The sum of the terms of each column vanishes. The matrix $\mathcal{A}$ is symmetric.

\section{Theorem 6.2.}

$$
\frac{1}{4(d-1)^{2}} \mathcal{A}_{l}^{l} \leq|l|^{2} \sum_{k}[\rho(k)]^{2}+\sum_{k}|k|^{2}[\rho(k)]^{2} .
$$

Proof. We have,

$$
\left|\mathcal{A}_{l}^{l}\right| \leq \sum_{k, s}\left\|\Gamma_{k, s}^{l}\right\|_{H S}^{2}[\rho(k)]^{2}
$$

which by $\left(2.4_{a}\right)$ is equal to

$$
\sum_{k}[\rho(k)]^{2}\left(\left\|b_{k, l-k}\right\|_{H S}^{2}+\left\|b_{k-l, l}\right\|_{H S}^{2}+\left\|b_{l+k, k}\right\|_{H S}^{2}\right)
$$

Finally, using the expression of $b_{k, j}$ we get the inequality $\left\|b_{k, j}\right\|_{H S}^{2} \leq(d-$ $1)^{2}\left(|k|^{2}+|j|^{2}\right)$.

\section{References}

1. H. Airault, P. Malliavin. Quasi-invariance of Brownian measures on the group of circle homeomorphisms and infinite-dimensional Riemannian geometry. $J$. Funct. Anal. 241 (1) (2006), 99-142.

2. V. I. Arnold. Sur la géométrie différentielle des groupes de Lie de dimension infinie et ses applications a l'hidrodynamique des fluides parfaits. Ann. Inst. Fourier 16 (1966), 316-361.

3. V. I. Arnold, B. A. Khesin. Topological Methods in Hydrodynamics. SpringerVerlag, New York, 1998.

4. A. B. Cruzeiro, F. Flandoli, P. Malliavin. Brownian motion on volume preserving diffeomorphisms group and existence of global solutions of $2 \mathrm{D}$ stochastic Euler equation. J. Funct. Anal. 242 (2007), 304-326.

5. D. Ebin, J. Marsden. Groups of diffeomorphisms and the motion of incompressible fluid. Ann. Math. 92 (2) (1970), 102-163. 
6. P. Malliavin. Stochastic Analysis. Grund. der Mathem. Wissen., SpringerVerlag, 1997. 